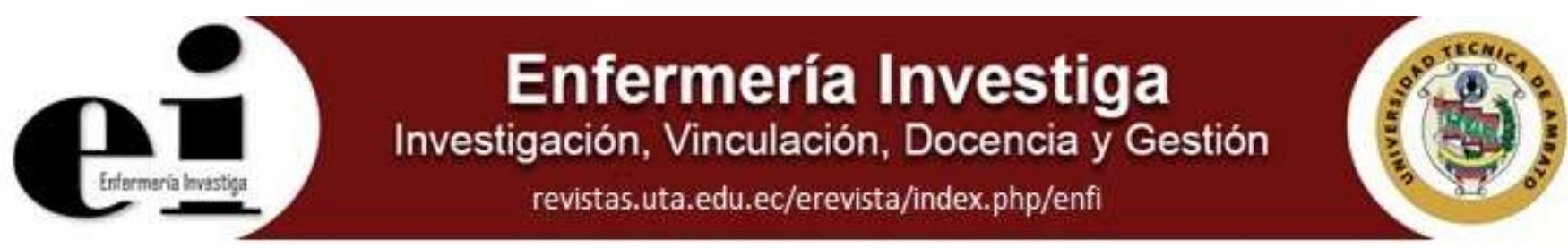

Editorial

\title{
Accidentes laborales en Enfermería
}

\author{
Amelia Patricia Panunzio R. ${ }^{1}$ \\ 1 Universidad del Zulia-Venezuela \\ Panunzio R. Amelia P., Accidentes laborales en Enfermería, Enferm Inv. 2020; 5(2):1-3
}

2477-9172 / 2550-6692 Derechos Reservados ( 2020 Universidad Técnica de Ambato, Carrera de Enfermería. Este es un artículo de acceso abierto distribuido bajo los términos de la Licencia Creative Commons, que permite uso ilimitado, distribución y reproducción en cualquier medio, siempre que la obra original es debidamente citada.

Historia:

Recibido: 21 enero 2020

Aceptado: 10 febrero 2020

Dentro del ámbito clínico asistencial el personal del área de enfermería al desempeñar sus actividades, se expone a factores de riesgos de diversa naturaleza, biológicos, químicos, físicos, mecánicos, disergonómicos y psicosociales, cuya dinámica determina la ocurrencia de enfermedades y accidentes de trabajo.

A propósito de accidentes laborales, estos son sucesos imprevistos que producen en el trabajador lesiones resultantes de una acción determinada en el curso o en ocasión del trabajo; son numerosos los factores que contribuyen en su aparición y pocas veces, por no decir ninguna, los accidentes ocupacionales son el resultado de una única causa o acción (1).

La exposición al riesgo de accidentes dentro del contexto clínico asistencial, no es la misma para todo el colectivo de trabajadores, sea en centros hospitalarios o extra hospitalarios, varía en función del tipo de trabajo desempeñado por cada categoría profesional, destacándose la mayor incidencia en el personal que presta asistencia sanitaria directa. La enfermería representa una de las ocupaciones dentro del sector sanitario que, en términos de exposición a riesgos ocupacionales, dominan entre las demás profesiones del área de la salud.

Un recorrido entre las numerosas evidencias científicas publicadas en la literatura reciente muestra un perfil de accidentalidad en profesionales y técnicos de la enfermería, muy variado, en los que destacan los siguientes : exposición accidental percutánea y mucocutánea a sangre y otros fluidos biológicos potencialmente infecciosos; lesiones por sobreesfuerzos físicos; resbalones y caídas; lesiones por violencia; traumas físicos por contacto con fuentes de energía; quemaduras térmicas o químicas; asfixia y lesiones por contacto accidental con agentes químicos tóxicos (2-9).

El accidente que continua observándose con la mayor prevalencia en enfermeras es el contacto percutáneo a sangre y otros fluidos biológicos ( pinchazos, cortaduras y laceraciones, con agujas y objetos punzo cortantes), seguido de contacto muco-cutáneo ( salpicaduras y splash de sangre o fluidos biológicos en membranas mucosas y piel no intacta); las lesiones musculo esqueléticas por sobre esfuerzo en la asistencia de pacientes, lesiones por resbalones, tropiezos y caídas y violencia verbal y física ocupan frecuencias que engrosan la tasa de accidentalidad en las enfermeras (2,4-5,8-10).

En relación a la exposición a sangre y fluidos corporales, es bien reconocido en entornos sanitarios como un peligro ocupacional que impacta a miles de trabajadores del cuidado de la salud por su potencial para transmitir patógenos sanguíneos, entre ellos, virus de la hepatitis $\mathrm{B}$ (VHB), hepatitis $\mathrm{C}(\mathrm{VHC})$ o virus de inmunodeficiencia humana (VIH), debido a contacto percutáneo y contacto de mucosas o piel no intacta, con sangre, tejidos u otros fluidos biológicos potencialmente infecciosos (11-12).

En el personal de enfermería la alta prevalencia de accidentes por exposición a sangre y fluidos biológicos donde está implicada la vía intradérmica (pinchazos, cortes, laceraciones) puede explicarse por varios factores relacionados; entre ellos, percepción del riesgo, factores propios de la asistencia directa al paciente, donde es común actividades de toma de muestra, sutura, inyecciones, entre otras, que involucran la manipulación frecuente de agujas, muchas veces desprotegidas, su desconexión de la jeringa, su re-encapuchado, inadecuado sistema para descarte de punzo cortantes, lo que aunado a factores que también son comunes, tales como, presión de 
tiempo, sobre carga de trabajo, trabajo por tunos, estrés, fatiga y cansancio determinan la ocurrencia de tal accidentalidad (2-4, 8,12-13).

Dentro de la problemática de exposición a riesgos ocupacionales, los trastornos musculoesqueléticos (TME) son considerados hoy en día, a nivel global, las lesiones profesionales más comunes y costosas y representan un tercio de las lesiones relacionadas con el trabajo cada año. La enfermería se encuentra entre las diez ocupaciones que causan lesiones musculoesqueléticas destacando a nivel lumbar la mayor prevalencia (14-15).

Factores organizacionales relacionados con la carga y contenido del trabajo, factores de riesgo disergonómico, posturas inadecuadas, movimientos de flexión, torsión, levantamiento y sobre esfuerzo físico en el manejo y movilización de pacientes, han sido determinantes en la ocurrencia de TME que contribuyen a disminuir la eficiencia del trabajo y aumentan las tasas de accidentes (10).

En efecto, el sobre esfuerzo físico es un evento frecuentemente reportado en la accidentalidad en enfermeras como causa de lesiones y enfermedades ocupacionales (6-7). Accidentes frecuentes son también las caídas, resbalones y tropiezos que pueden ser la causa de esguinces, distensiones y desgarros musculares en las enfermeras, citándose el esfuerzo excesivo como uno de los factores determinantes $(6-7,9)$.

Dentro de este panorama, un riesgo que genera gran preocupación en el ámbito de la asistencia sanitaria, es la violencia, problemática que en los últimos años va en aumento considerable en los trabajadores de la salud en todo el mundo. La Violencia en el entorno ocupacional se define como los eventos que ocurren en el lugar de trabajo y durante el trabajo y que causan lesiones físicas o mentales, razón por la cual, los casos de violencia se consideran accidentes laborales $(2,4,9)$.

La violencia verbal y física es origen de lesiones que son reportadas comúnmente en la accidentalidad de este grupo laboral, destacando particularmente los departamentos de emergencia como uno de los entornos de más alto riesgo $(4,9,16)$.

En la magnitud y trascendencia de la exposición a riesgos ocupacionales en el sector sanitario, especial mención merecen los riesgos psicosociales que suponen a nivel global, más de un tercio de los accidentes y enfermedades derivadas del trabajo y un $17 \%$ de las bajas laborales (17).

Tal situación la vemos reflejada en el contexto laboral de los profesionales de Enfermería, donde se evidencian problemáticas relacionadas con condiciones laborales marcadas por la presencia de factores de riesgos de tipo psicosocial, entre ellos, responsabilidades excesivas, sobrecarga de trabajo, largas jornadas de trabajo, turnos rotatorios, trabajo nocturno, frecuentes cambios de servicios y carga psicológica por manejo de situaciones críticas, conflictos entre superiores y colegas, etc., que determinan la presencia de estrés, fatiga y burnout, reconocidos predictores de consecuencias físicas y mentales en el trabajador que influencian la ocurrencia de accidentes ocupacionales $(13,15-18)$.

En efecto, en diversos estudios sobre prevalencia de accidentes en enfermería, se encuentra frecuentemente la asociación con factores de riesgos de tipo psicosocial, tales como, trabajo por turnos, turnos nocturnos, largas jornadas de trabajo, presiones laborales, desordenes del sueño, depresión (2, 4,8-9,13,18).

La somnolencia y la fatiga son consecuencias comunes en trabajadores que desempeñan largas jornadas de trabajo y turnos nocturnos y factores de riesgo bien conocidos para la ocurrencia de accidentes. La alteración del sueño causada por las demandas profesionales contribuye a disminuir la eficiencia del trabajo, aumenta las tasas de accidentes y disminuye la función cognitiva causada por la falta de sueño (13).

A la luz de las evidencias se nos muestra que en las enfermeras continúan observándose altos niveles de lesiones y enfermedades relacionadas con el trabajo, el rango de los riesgos es muy amplio y la dinámica de exposición a factores de diversa naturaleza es compleja ; las consecuencias, pueden ser de diversa gravedad, muchas pueden incluir discapacidad y muerte; sin embargo, el impacto no solo es en la salud del personal, es también en el rendimiento y satisfacción en el trabajo, seguridad del paciente y aumento de los costos en salud.

Determinar las causas inmediatas y básicas seguirá siendo fundamental para lograr intervenciones efectivas que contribuyan a la prevención y control de riesgos y peligros que impactan en la salud y seguridad de este importante recurso humano en el contexto sanitario.

\section{Referencias}

1. Organización Internacional del Trabajo. Enciclopedia de Salud y Seguridad en el Trabajo Volumen VIII Prevención de Accidentes Capitulo 56: Accidentes y Gestión de la Seguridad. 4th Edición Ginebra 2014. https://labordoc.ilo.org/permalink/41ILO_INST/kol415/alma994880063402676

2. Kowalczyk A, Kulczycka K, Stychno E, Chilimoniuk B. Characteristics of occupational hazards at the workplace of a nurse. Journal of Education, Health and Sport. 2018;8(9):1328-1337.

3. D'Ettorre G. Needlestick and Sharp Injuries Among Registered Nurses: A Case-Control Study. Ann Work Expo Health. 2017 Jun 1;61(5):596-599.

4. Çelikkalp Ü, Dilek F. Factors affecting the occupational accident rates among nurses. Rev Esc Enferm USP. 2019 Dec 2;53:e 03524.

5. Joshua R, Karkada S. A Review on Occupational Health Hazards and its Consequences among Nurses International Journal of Nursing Research and Practice 2017 Vol. 4 No. 2 July -December. 
6. Dressner M, Kissinger, S. Occupational injuries and illness among registered nurses. Monthly Labor Review, U.S. Bureau of Labour Statistic, November 2018

7. Dressner M. Hospital workers: An assessment of occupational injuries and illness, Monthly Labor Review, , U.S. Bureau of Labour Statistic, June 2017

8. Bagheri Hosseinabadi M, Khanjani N, Etemadinezhad S, Samaei SE, Raadabadi M,Mostafaee M. The associations of workload, individual and organisational factors on nurses' occupational injuries. J Clin Nurs. 2019 Mar;28(5-6):902-911.

9. Unruh L, Asi Y. Determinants of Workplace Injuries and Violence Among Newly Licensed RNs. Workplace Health Saf. 2018 Oct;66(10):482-492.

10. Richardson A, Mc Noe B, Derrett S, Harcombe H. Interventions to prevent and reduce the impact of musculoskeletal injuries among nurses: A systematic review. Int J Nurs Stud. 2018; Jun;82:58-67.

11. Gholami A, Borji A, Lotfabadi P, Asghari A. Risk factors of needlestickand sharps injuries among healthcare workers. Int J Hosp Res. $2013 ; 2(1): 31$ e38.

12. Moralejo D, El Dib R, Prata RA, Barretti P, Corrêa I. Improving adherence to Standard Precautions for the control of health care-associated infections. Cochrane Database Syst Rev. 2018; Feb 26;2:CD010768

13. Leyva-Vela B, Jesús Llorente-Cantarero F, Henarejos-Alarcón S, Martínez-Rodríguez A. Psychosocial and physiological risks of shift work in nurses: a cross-sectional study. Cent Eur J Public Health. 2018; Sep;26(3):183-189

14. Tosunoz IK, Oztunc G. Low Back Pain in Nurses. International Journal. 2017 Sep;10(3):1728.

15. Bernal D, Campos-Serna J, Tobias A, Vargas-Prada S, Benavides FG, Serra C.Work-related psychosocial risk factors and musculoskeletal disorders in hospital nurses and nursing aides: a systematic review and meta-analysis. Int J Nurs Stud.2015 Feb;52(2):635-48.

16. Ramacciati, N., Ceccagnoli, A., Addey, B., Lumini, E., \& Rasero, L. (2018). Violence towards emergency nurses: A narrative review of theories and frameworks. International Emergency Nursing 2018; 39, 2-12

17. Vidal-Blanco G, Oliver A, Galiana L, Sansó N. Quality of work life and self-care in nursing staff with high emotional demand. Enferm Clin. 2019; May Jun;29(3):186-194

18. Salvagioni DAJ, Melanda FN, Mesas AE, González AD, Gabani FL, Andrade SM. Physical, psychological and occupational consequences of job burnout: A systematic review of prospective studies. PLoS One. 2017; Oct 4;12(10):e0185781

Enferm Inv. 5(2):1-3 